\title{
O Mestrado Profissional em Informática na Educação do IFRS: Temas de Pesquisa, Objetos de Investigação e Produtos Privilegiados
}

\author{
Julian Silveira Diogo de Ávila Fontoura \\ Universidade do Vale do Rio dos Sinos - UNISINOS \\ julian.diogo@gmail.com \\ Josiane Carolina Soares Ramos Procasko \\ Instituto Federal de Educação, Ciência e Tecnologia do Rio Grande do Sul - IFRS \\ josiane.ramos@poa.ifrs.edu.br
}

\begin{abstract}
Resumo: O estudo aqui apresentado busca identificar, a partir das dissertações produzidas junto ao Mestrado Profissional em Informática na Educação do Instituto Federal de Educação, Ciência e Tecnologia do Rio Grande do Sul (IFRS), os temas de estudo presentes nas investigações, os objetos de pesquisa privilegiados e os "produtos" desenvolvidos junto à produção acadêmica do curso. Para isto, nos utilizamos - como estratégia metodológica - dos "princípios" de Estado de Conhecimento, caracterizados pelo foco na pesquisa de caráter histórico-bibliográfico, exploratório-investigativo, inventariante e descritivo. O corpus de análise se apresenta a partir das 21 dissertações produzidas pelas 2 (duas) primeiras turmas do curso. Os resultados desta investigação apontam para a complexidade e diversidade presente junto aos estudos do campo da Informática na Educação, especialmente, quando articulados a Educação Profissional, Científica e Tecnológica. No Mestrado Profissional em Informática na Educação do IFRS, destacam-se temas relacionados as "tecnologias e as aprendizagens"; nos objetos de pesquisa percebemos uma predileção as "práticas pedagógicas" no ambiente escolar; e como produtos fruto destas investigações, evidenciamos uma relação direta com o desenvolvimento de "matrizes e protocolos" de ação para o campo educacional.
\end{abstract}

Palavras-Chave: mestrado profissional, produção do conhecimento, informática na educação.

\section{IFRS Professional Master in Informatics in Education: Research Themes, Research Objects and Privileged Products}

\begin{abstract}
The study presented here seeks to identify, from the dissertations produced with the Professional Master in Informatics in Education of the Federal Institute of Education, Science and Technology of Rio Grande do Sul (IFRS), the study themes present in the investigations, the objects privileged research projects and the "products" developed along with the academic production of the course. For this, we use - as methodological strategy - the principles of State of Knowledge characterized by the focus on the research of historical-bibliographical character, exploratory research, inventive and descriptive. The corpus of analysis is presented from the 21 dissertations produced by the first two (2) groups of students of the course so far. The results of this study point to the complexity and diversity present in studies of the field of Informatics in Education, especially when articulated to Vocational, Scientific and Technological Education. Along with the Professional Master in Informatics in Education of IFRS, we highlight those themes related to "technologies and learning"; In the research objects we noticed a predilection for the "pedagogical practices" in the school environment; and as products, those related to the development of "matrices and protocols" for the educational field.
\end{abstract}

Keyword: professional master, knowledge production, informatics in education.

\section{Introdução}

A gênese deste estudo se insere em um contexto de profundas transformações na forma de produção e regulação do conhecimento científico, especialmente aquele produzido dentro do espaço universitário (Oliveira; Fernandes, 2017), repleto de influências de agentes internos e externos, como os organismos internacionais/multilaterais, as agências reguladoras nacionais, os processos de 
globalização, o avanço das tecnologias digitais de informação e comunicação, além das novas "formas de ser e de estar" no mundo contemporâneo. Dessa forma, o espaço universitário se apresenta como um elemento fundamental na produção de conhecimentos, já que se constitui como um espaço privilegiado para este fim (Severino, 2002), que posteriormente serão capilarizados no campo social, e (re)significado muitas vezes pelas comunidades de pesquisa.

A Informática na Educação, como campo formativo, tornou-se objeto de busca de vários profissionais, não só apenas daqueles da área da educação, mas também aqueles "migrantes" de outros campos disciplinares do conhecimento. Este movimento não de consubstancia apenas no sentido da instrumentalização destes sujeitos, mas como uma possibilidade efetiva na resolução de problemáticas presentes nos distintos espaços educativos (formais e não-formais), tendo na informática e suas tecnologias a ferramenta para isso. É imperativo para a investigação científica compreender a forma como o conhecimento acadêmico produzido no interior desses cursos se apresenta, buscando entender os mecanismos estabelecidos e operacionalizados nesta produção.

A investigação aqui apresentada, busca indicar a forma como o conhecimento no campo da Informática na Educação é consubstanciado frente ao curso de Mestrado Profissional em Informática na Educação do Instituto Federal de Educação, Ciência e Tecnologia - Campus Porto Alegre, a partir dos temas de investigação, objetos de pesquisa e produtos privilegiados por esta comunidade de pesquisa. Como metodologia na produção dos dados, nos utilizamos dos "princípios" de Estado do Conhecimento, na perspectiva de Morosini (2015), com foco na pesquisa de caráter histórico-bibliográfico (Fiorentini; Lorenzato, 2007), exploratório-investigativo (Melo, 2006), inventariante e descritivo (Ferreira, 2002). O material bibliográfico utilizado neste estudo, se constitui das dissertações dos egressos do curso stricto sensu de Mestrado Profissional em Informática na Educação. No processo de delimitação da pesquisa, utilizamos todas as dissertações defendidas desde a sua criação/implementação em 2015 até o ano de 2018.

\section{Caminho Metodológico}

Em função da natureza do estudo aqui apresentado, compreendemos que o processo metodológico escolhido acaba ocupando um lugar de destaque, da mesma forma que Vieira (1992, p. 29), percebemos a metodologia como uma "trajetória teórica", um percurso realizado através da articulação de um conjunto de conceitos utilizados tanto na produção dos dados, quanto na análise de um objeto. Dessa forma, evidenciamos a importância da escolha metodológica dentro do processo investigativo como um fator de extrema relevância "metodologias distintas resultam em diferentes resultados, acarretando diferentes entendimentos sobre a problemática investigada" (Fontoura; Morosini, 2017, p. 143).

As pesquisas conhecidas como "Estado do Conhecimento" ou ainda "Estado da Arte", para alguns, são caracterizadas, pela sua proximidade com o caráter bibliográfico e o seu compromisso no mapeamento (identificação) da produção acadêmica em diferentes campos do saber. Na busca pela compreensão de aspectos e dimensões de determinado campo científico a partir de diferentes materiais bibliográficos, como dissertações de mestrado, teses de doutorado, publicações em periódicos, entre outros tantos formatos utilizados para a difusão do conhecimento acadêmico (Ferreira, 2002, p. 257). Para Morosini (2015), os Estados de Conhecimento acabam se apresentando como "um momento ímpar para os pesquisadores, possibilitando um contato aprofundado com a produção acadêmica de distintas comunidades de pesquisa sob uma determinada 
temática, a partir da sua produção bibliográfica", já que permite ao pesquisador a "identificação, registro, categorização que levem à reflexão e síntese sobre a produção científica de uma determinada área, em um determinado espaço de tempo" (Morosini, 2015, p. 102).

Nesta perspectiva, a construção do Estado do Conhecimento se estabelece a partir de três fases metodológicas distintas: a Bibliografia Anotada como um primeiro movimento de aproximação com o material bibliográfico a ser analisado; a Bibliografia Sistematizada como sendo um momento de aprofundamento maior junto ao material bibliográfico captado, tendo em vista os objetivos do estudo; e a Bibliografia Categorizada que culmina neste processo com a reorganização do material bibliográfico analisado (corpus de análise), a partir de categorias analíticas (a priori ou a posteriori), no sentido de "traduzir" o que dizem os trabalhos que compõem o corpus construído.

No desenvolvimento das fases metodológicas, ocorre um intendo processo de impregnação junto ao material a ser analisado, um envolvimento entre o pesquisador e o corpus de análise, “(...) a impregnação é condição para um trabalho criativo e original. A partir do envolvimento aprofundado é que se criam condições de emergência autoorganizada das novas compreensões", como coloca Moraes e Galiazzi (2006, p. 121). Desta forma, as pesquisas do Estado de Conhecimento podem ser caracterizadas, de acordo com o processo de constituição e análise dos dados, em especial pelo seu caráter histórico-bibliográfico (Fiorentini; Lorenzato, 2007), em sua abordagem exploratóriainvestigativa (Melo, 2006), inventariante e descritiva (Ferreira, 2002).

\section{Os Achados de Pesquisa: Compreendendo o Campo de investigação}

No universo qual se apresentam os estudos junto ao material bibliográfico analisado, temos como temática de pesquisa a Informática na Educação, como a grande categoria que acaba por unir todos os trabalhos presentes, porém, quando nos referimos ao tema de pesquisa, estamos falando de uma unidade de sentido menor, específica e articulável a temática, da mesma forma que Lakatos e Marconi (2006, p. 126) compreendemos o tema de pesquisa como sendo o "o assunto que se deseja provar ou desenvolver". Dessa forma, a partir de nossas análises, emergem junto ao material bibliográfico, três categorias (tabela 1) que tem nos processos tecnológicos e sua relação com a sociedade seu cerne.

Tabela 1. Temas de pesquisa presentes nas dissertações do Mestrado Profissional em Informática na Educação do Instituto Federal de Educação, Ciência e Tecnologia.

\begin{tabular}{|c|c|}
\hline CATEGORIAS & TEMAS DE PESQUISA \\
\hline \multirow{8}{*}{$\begin{array}{l}\text { TECNOLOGIAS E } \\
\text { APRENDIZAGENS }\end{array}$} & Ambientes Virtuais de Aprendizagem \\
\hline & Gameficação \\
\hline & Processos de Ensino e Aprendizagem \\
\hline & Jogos Digitais \\
\hline & Objetos de Aprendizagem e Sala de Aula Invertida \\
\hline & Movimento Maker \\
\hline & Robótica Educacional \\
\hline & Design Thinking \\
\hline \multirow{5}{*}{$\begin{array}{c}\text { TECNOLOGIAS E PROCESSOS } \\
\text { DE FORMAÇÃO }\end{array}$} & Atividades Formativas Discentes \\
\hline & Formação Continuada \\
\hline & Práticas Pedagógicas \\
\hline & Educação a Distância \\
\hline & Pesquisa Científica \\
\hline
\end{tabular}

V. $17 \mathrm{~N}^{\mathrm{o}}$ 3, dezembro, 2019 RENOTE DOI: 
TECNOLOGIAS, POLÍTICA

E GESTÃOO
Política Educacional

Gestão Educacional

Na categoria Tecnologias e Aprendizagens aglutinados uma série de estudos relacionados as ferramentas digitais, o pensamento computacional e a potência das tecnologias frente aos processos de aprendizagem, a partir dos ambientes virtuais de aprendizagem, a gameficação, os jogos digitais, os objetos de aprendizagem e sala de aula invertida, o movimento maker, a robótica educacional e o design thinking. $\mathrm{Na}$ categoria Tecnologias e Processos de Formação temos reunidos estudos que percebem as tecnologias como elemento importante junto aos processos de formação discente e docente, nos diferentes níveis educacionais, a luz das Atividades Formativas Discentes, a Formação Continuada, as Práticas Pedagógicas, a Educação a Distância, e a produção da Pesquisa Científica. Pôr fim, junto a categoria Tecnologias, Políticas e Gestão, temos refletida a preocupação de alguns autores em compreender os processos de implementação das políticas educacionais e práticas de gestão educacional que focalizam seus esforços sob o viés da tecnologia.

Ao nos referirmos aos objetos de pesquisa, precisamos ter a clareza que os mesmos não são estruturas prontas, sólidas e imutáveis. Constituem-se sob um intenso processo dialético articulado a temática de estudo e ao tema a ser investigado, Eco (1995, p. 21) ao se referir sobre este processo de construção, destaca que "definir o objeto [de pesquisa] significa definir as condições sob as quais podemos falar, com base em certas regras que estabeleceram antes de nós", ou seja, a construção do objeto de pesquisa pressupõe, não apenas o conhecimento prévio sobre o que se deseja pesquisar, mas também, o domínio de técnicas que garantirão o sucesso nesta trajetória metodológica.

Dessa forma, os objetos de pesquisa estão diretamente relacionados com o foco do estudo, o eixo central da investigação, não podemos confundi-lo como sendo o assunto a ser desenvolvido, mas sim como uma parte integrante deste assunto. A variedade de objetos é uma das características marcadas em nossas análises, especialmente pela forma como os autores acabaram por relacionar seus estudos, as problemáticas cotidianas vividas e experienciadas nos diferentes espaços escolares que atuam. A partir do processo de categorização (Moraes; Galiazzi, 2007), reorganizamos os objetos de pesquisa das dissertações do Mestrado Profissional em Informática na Educação do IFRS e chegamos a três grandes categorias de análise: Práticas Pedagógicas; Instrumentalização em Tecnologias de Informação Digital $e$ Comunicação; e Gestão das Tecnologias Digitais (tabela 2).

Tabela 2. Objetos de pesquisa presentes nas dissertações do Mestrado Profissional em Informática na Educação do Instituto Federal de Educação, Ciência e Tecnologia.

\begin{tabular}{c|l}
\hline \multicolumn{1}{c|}{ CATEGORIAS } & \multicolumn{1}{c}{ OBJETOS DE PESQUISA } \\
\hline \multirow{4}{*}{$\begin{array}{c}\text { INFORMÁTICA NA EDUCAÇÃO } \\
\text { PRÁTICASPEDAGÓGICAS }\end{array}$} & Cursos de Extensão EAD \\
\cline { 2 - 2 } & Cursos Técnicos EAD \\
\cline { 2 - 2 } & Educação Empreendedora \\
\cline { 2 - 2 } & História e Cultura Afro-Brasileira no Currículo Escolar \\
\cline { 2 - 2 } & Inclusão \\
\cline { 2 - 2 } & Iniciação Científica no Ensino Médio \\
\cline { 2 - 2 } & Práticas Pedagógicas Docentes \\
\hline INFORMÁTICA NA EDUCAÇÃO - & Competência Informacional \\
\hline
\end{tabular}

V. $17 \mathrm{~N}^{\mathrm{o}}$ 3, dezembro, 2019 DOI: 


\begin{tabular}{c|l}
\hline INSTRUMENTALIZAÇÃO EM & Inserção das TIC's nos Anos Finais do Ensino Fundamental \\
TECNOLOGIAS DE INFORMAÇÃO & Processos de Formação Continuada em TIC's \\
\cline { 2 - 2 } $\begin{array}{c}\text { DIGAL E COMUNICAÇÃO } \\
\text { GESTÃO DAS }\end{array}$ & Robótica na Educação Infantil \\
\hline \multirow{2}{*}{$\begin{array}{c}\text { INFORMÁTICA NA EDUCAC̃O }- \\
\text { TECNOLOGIASDIGITAIS }\end{array}$} & Política Educacional de Tecnologias no Município de Canoas/RS \\
\cline { 2 - 2 } & Sistemas de Gestão \\
\hline
\end{tabular}

A categoria Informática na Educação - Práticas Pedagógicas, baseia-se na compreensão de que esta ação não pode ser reduzida à questão didática ou às metodologias de estudar e de aprender, como aponta Fernandes (2008, p. 159), mas "articulada à educação como prática social e ao conhecimento como produção histórica e social, numa relação dialética entre prática-teoria, conteúdo-forma e perspectivas interdisciplinares" (Fernandes, 2008, p. 159). Temos como objetos privilegiados de pesquisa os cursos de extensão EAD, os cursos técnicos EAD, a educação empreendedora, os processos de ensino e aprendizagem, a história e cultura afrobrasileira no currículo escolar, a inclusão, a iniciação científica no ensino médio e as práticas pedagógicas desenvolvidas por docentes.

O entendimento da categoria Informática na Educação - Instrumentalização em Tecnologias de Informação Digital e Comunicação, refletem a a importância das TIC's junto aos processos educativos e a adesão às novas tecnologias. Esse movimento é efetivado a partir de espaços de formação que reestruturam seu fazer pedagógico e da instrumentalização dos sujeitos da escola (professores, alunos e membros da comunidade escolar). Dessa forma, teremos uma possibilidade de estímulo à reflexão "sobre o seu papel no processo de ensino e aprendizagem frente a uma sociedade tecnológica", e fazendo do uso das tecnologias uma potência junto aos processos de aprendizagem, como aponta Belloni (2009, p. 24). Nesta categoria, temos evidenciados como objetos de pesquisa a competência informacional, a inserção das TIC's nos anos finais do ensino fundamental, os processos de formação continuada em TIC's, e a robótica na educação infantil.

Já a categoria Informática na Educação - Gestão das Tecnologias Digitais se apresenta a partir da leitura de Alves (2014) sobre o tempo e a inserção das tecnologias digitais nos espaços educativos. Esta categoria acaba por traduzir a operacionalização da implementação, articulação e desenvolvimento de tecnologias digitais com foco nos processos de gestão, emerge assim a missão de integrar as tecnologias digitais às práticas de gestão da educação. Alicerçados na categorização construída, constata-se que os objetos de pesquisa presentes no corpus de análise, abrangem diferentes dimensões no interior do campo educacional. Essas múltiplas dimensões demonstram a complexidade do ato educativo, materializando as distintas relações constituídas na educação em tempos de cibercultura, especialmente relacionados a gestão das tecnologias nas escolas estaduais de Porto Alegre/RS, a política educacional de tecnologias no município de Canoas/RS e aos sistemas de gestão.

O Mestrado Profissional se constitui como uma modalidade de Pós-Graduação stricto sensu voltada para a capacitação de profissionais, de forma a possibilitar a resolução de problemáticas relacionadas às demandas do mundo do trabalho, prioriza o atendimento aos profissionais de diversos setores que estejam trabalhando na área de estudo, com o intuito de promover melhor articulação entre universidade e a sociedade (Niezer et al., 2015). Para a obtenção do título de Mestre o discente além de ter o aproveitamento nos créditos das disciplinas obrigatórias e optativas, necessita produzir uma dissertação e um produto, na forma de complemento a pesquisa realizada, 
considerando os fundamentos teóricos seguros das tecnologias educacionais e dos fundamentos da educação (IFRS, 2014).

Dessa forma, os produtos das dissertações relacionam-se diretamente com o campo de atuação profissional dos discentes, alinhados a temática da Informática na Educação e ao tema de pesquisa anteriormente evidenciado. Os produtos possuem diferentes naturezas: objetos de aprendizagens, protocolos de avaliação de cursos, planos estratégicos, jogos digitais, cursos de formação entre outros. No sentido de dinamizar a apresentação destes produtos, reunimos os mesmos a partir de unidades de sentido (Moraes; Galiazzi, 2007) a partir da aglutinação das suas similaridades, e aplicabilidade, como demonstra na tabela abaixo (tabela 3).

Tabela 3. Produtos de pesquisa presentes nas dissertações do Mestrado Profissional em Informática na Educação do Instituto Federal de Educação, Ciência e Tecnologia.

\begin{tabular}{|c|c|}
\hline CATEGORIAS & PRODUTOS \\
\hline \multirow{9}{*}{$\begin{array}{l}\text { INFORMÁTICA NA EDUCAÇÃO - } \\
\text { MATRIZES E PROTOCOLOS }\end{array}$} & Avaliação de Massive Open Online Course (MOOCS) \\
\hline & Avaliação e Planejamento de Cursos Técnicos em EAD \\
\hline & Orientações para a Implementação de Sistemas de Gestão \\
\hline & Mapeamento de Políticas Educacionais \\
\hline & $\begin{array}{l}\text { Conjunto de Critérios para Sala de Aula Invertida no Ensino de } \\
\text { Inglês }\end{array}$ \\
\hline & Cinco Passos para a Aprendizagem Criativa \\
\hline & $\begin{array}{l}\text { Plano Estratégico de Consolidação da Tecnologia no Ambiente } \\
\text { Escolar }\end{array}$ \\
\hline & $\begin{array}{l}\text { Critérios para Elaboração de Jogos Digitais Educacionais para } \\
\text { Transtorno do Espectro Autista }\end{array}$ \\
\hline & $\begin{array}{l}\text { Protocolo Didático de Formação Continuada para a Inserção das } \\
\text { TICs }\end{array}$ \\
\hline \multirow{8}{*}{$\begin{array}{l}\text { INFORMÁTICA NA EDUCAÇÃO - } \\
\text { FERRAMENTAS DIGITAIS }\end{array}$} & Alphabetiz@r \\
\hline & Objeto de Aprendizagem \\
\hline & Jogo de Tabuleiro \\
\hline & Cachorro Robô \\
\hline & El Mochilero \\
\hline & Gerenciador de Apresentação \\
\hline & Descrimagem \\
\hline & CONSPROG \\
\hline \multirow{4}{*}{$\begin{array}{c}\text { INFORMÁTICA NA EDUCAÇÃO - } \\
\text { PLATAFORMAS DIGITAIS E } \\
\text { FORMAÇÃO }\end{array}$} & Plataforma Box Plus \\
\hline & Sistema Compartilhar \\
\hline & Portfólio Digital Interativo \\
\hline & Curso Linux Educacional \\
\hline
\end{tabular}

A categoria Informática na Educação - Matrizes e Protocolos, reúne produtos de dissertações que partilham da proposição de elaboração de documentos, diretrizes, critérios, modelos avaliativos e planos estratégicos que servem como instrumentos para gestores, professores e instituições de ensino nas soluções de dificuldades do contexto escolar, especificamente no que se refere a questões estruturais do espaço educativo. Balizando esta categoria, nos apoiamos nas reflexões de Dallacosta, Cazetta e Souza (2010), sobre a importância da produção de materiais instrucionais no contexto das novas tecnologias.

A categoria Informática na Educação - Ferramentas Digitais, estuda o desenvolvimento de aplicativos, softwares, jogos e instrumentos digitais que possuem potencial para distintos processos de aprendizagem, além de articulados e experienciados no espaço escolar. Para esta compreensão, nos valemos dos estudos de Sartori e Soares, (2013, p. 12), ao refletirem sobre as ferramentas digitais e seus impactos no mundo contemporâneo "em que é preciso educar numa sociedade em que

V. $17 \mathrm{~N}^{\mathrm{o}}$ 3, dezembro, 2019

RENOTE DOI: 
os dispositivos tecnológicos e midiáticos produzem outras sensibilidades, deslocalizam o saber, inauguram novas formas de expressão, Comunicação e Educação".

E a categoria Informática na Educação - Plataformas Digitais e Formação reúne produtos que buscam articular a prática pedagógica com o desenvolvimento de espaços (sistemas, portfólios e plataformas) virtuais que potencializem os processos desenvolvidos no ambiente educativo. Almeida (2003, p. 201) nos auxilia neste entendimento, ao perceber as plataformas digitais como possibilidade de "suportar tanto as tradicionais formas mecanicistas de transmitir conteúdos digitalizados como processos de produção colaborativa de conhecimento". Sobre essa reflexão, cabe frisar a relevância da superação de formas tradicionais de educação (modelos), buscando verdadeiramente a constituição de novas formas de se relacionar com o mundo multifacetado do ciberespaço.

\section{Algumas Considerações}

O estudo aqui apresentado se mostra como um primeiro e importante movimento de compreensão do entendimento da produção de conhecimento do curso de Mestrado Profissional em Informática na Educação do IFRS. Importante destacarmos que este movimento alinha-se aos desafios das demandas legais advindas Lei Federal $\mathrm{n}^{\mathbf{o}}$ 11.892/2008 que concebeu os Institutos Federais de Educação, Ciência e Tecnologia; dos órgãos nacionais de regulação da Pós-Graduação; e da proposta de formação presente no PPC do curso. A modalidade profissional procura responder a busca por uma capacitação profissional diferenciada, constituindo-se como uma alternativa efetiva à formação tradicional da pós-graduação stricto sensu.

Estudos já apontam para a forma interdisciplinar que os conhecimentos no campo da Informática da Educação são produzidos, a investigação aqui apresentada acaba por corroborar essa afirmação, já que as dissertações que compõem as análises, emergem em um cenário complexo, onde uma série de saberes são mobilizados na tentativa de interpretação de realidades distintas (problemas de pesquisa). Essa forma "interdisciplinar" de articulação, faz com que o campo de estudos no qual a Informática na Educação se manifeste, seja repleto que influências de outros campos disciplinares do conhecimento, como podemos perceber ao observarmos as relações estabelecidas pelos "jovens pesquisadores" em suas dissertações.

No que se refere aos temas de pesquisa, as investigações que fazem parte do corpus de análise, acabam por evidenciar três movimentos de compreensão envolvendo a Informática na Educação: um com foco no entendimento das Tecnologias $e$ as Aprendizagens, Tecnologias e Processos de Formação, e Tecnologias, Políticas e Gestão. O primeiro destes acaba se destacando no seu quantitativo em relação aos demais, cerca de $47,6 \%$ de toda a produção acadêmica componente do corpus de análise, com foco na Gameficação. A diversidade de objetos de pesquisa que se mostram no conjunto das dissertações analisadas, acabam por desvelar as Práticas Pedagógicas como sendo o grupo de objetos de investigação privilegiado por esta comunidade de pesquisa (cerca de 57\%) com foco centralizado nos processos de Ensino e Aprendizagem.

Os produtos no contexto do Mestrado Profissional acabam por muitas vezes representar a materialização da resolução da problemática de pesquisa, ou melhor, a materialidade do estudo. Dentro desta perspectiva, os produtos produzidos pelos "jovens pesquisadores", se efetivam a partir de três dimensões: as Ferramentas Digitais; as Plataformas Digitais e Formação e ainda Matrizes e Protocolos. Sendo que destes, 
majoritariamente, temos nas Matrizes e Protocolos o maior foco na produção, onde os produtos se mostram a partir de guias, roteiros ou ainda estratégias de ação.

A partir dos dados produzidos com esta investigação, pretendemos fazer um acompanhamento sistêmico sob os caminhos/trajetórias de pesquisa trilhadas pelos "jovens pesquisadores" pertencentes a esta comunidade de pesquisa, de forma a evidenciar os diferentes movimentos investigativos desenvolvidos e estabelecidos junto ao Mestrado Profissional em Informática na Educação. Compreendemos ainda que a utilização de estudos caracterizados como sendo de Estado do Conhecimento se apresentam como fundamentais para o desenvolvimento das pesquisas nos distintos campos do saber. Esta abordagem metodológica permite a construção de um panorama, ou ainda, uma perspectiva de entendimento do campo de pesquisa, especialmente quando se busca compreender os processos de produção de conhecimento por diferentes comunidades de pesquisa, que em seu conjunto, acabam por "desenhar" o pensamento do campo científico de determinado campo do saber.

Temas de investigação, objetos de estudo e produtos se mostram de forma interrelacionada no contexto do curso de Mestrado Profissional em Informática na Educação do IFRS, imbricando-se diretamente com a atividade profissional dos "jovens pesquisadores". Em alguma medida, este conjunto acaba por compor um quadro interessante aos investigarmos esta comunidade de pesquisa, podemos ainda, com estes dados, traçar uma espécie de "perfil" do curso. Esta leitura sobre a produção do conhecimento no contexto deste curso foi um primeiro exercício de entendimento das possibilidades e formas perceber a Informática na Educação sob a perspectiva da Educação Profissional e Tecnológica, alinhada a Educação Superior em um contexto de fomento a política pública como é o caso dos Institutos Federais.

A diversidade presente nos temas, objetos e produtos, demonstram ainda como o campo da Informática na Educação tem seu corpus constituído de forma interdisciplinar, reunindo em seu bojo um conjunto de saberes e conhecimentos próprios com fronteiras muito fluidas, possibilitando uma série de relações com outros campos e saberes disciplinares. A Informática na Educação, emerge para além de uma "ferramenta", mas sim como uma possibilidade real de transformação da sociedade em um contexto extremamente mutável como o que experienciamos na contemporaneidade.

\section{Referências}

ALMEIDA, M. E. B. Redesenhando estratégias na própria ação: Formação do professor a distância em ambiente digital. In: Valente, J. A. et al. Educação a distância via internet. São Paulo: Avercamp, 2003.

ALVES, R. M. Gestão educacional e novas tecnologias da informação e comunicação: atualizações necessárias disponíveis para a cultura educacional. Revista e-Curriculum, São Paulo, n. 12, v. 2 mai/out. 2014. Disponível em: 〈http://bit.ly/2YV9IHg〉. Acesso em: 10 set 2019.

BELLONI, M. L. O que é mídia-educação? Campinas, SP: Autores Associados, 2009.

DALlACOSTA, A.; CAZETTA, G.; SOUZA, S. G. DE. Novas tecnologias aplicadas na elaboração de material instrucional online. In: $3^{\circ}$ Simpósio Hipertexto e Tecnologias na Educação - Redes Sociais e Aprendizagem. Anais Eletrônicos. 2010. Disponível em: <http://bit.ly/30ztpVk>. Acesso em 05 set 2019. 
ECO, U. Como se faz uma tese. 12. ed. Tradução de Gilson César Cardoso Sousa. São Paulo: Perspectiva, 1995.

FERNANDES, A. Mestrado profissional - algumas reflexões. Oculum ensaios: revista de arquitetura e urbanismo - n. 4, pp. 106-109. 2005. Disponível em: <http://bit.ly/30INdpF>. Acesso em 02 set 2019.

FERREIRA, N. S. de. A. As Pesquisas Denominadas "Estado da Arte". Educação \& Sociedade, ano XXIII, n. 79, ago, 2002. Disponível em: <http://bit.ly/2O6XI4i>. Avesso em 10 set 2019.

FIORENTINI, D.; LORENZATO, S. Investigação em Educação Matemática: Percursos Teóricos e Metodológicos. 2 ed. Campinas: Autores Associados. 2007.

FONTOURA, J.; MOROSINI, M. A Gestão da Educação Superior no Contexto da Educação, Profissional e Tecnológica: Os Diferentes Focos na Pesquisa. Revista Brasileira da Educação Profissional e Tecnológica. v. $2 \mathrm{n}^{\circ}$ 13, p. 137-149. 2017. Disponível em: 〈http://bit.ly/2KWwb2p>. Acesso em 12 jun 2019.

IFRS - Instituto Federal de Educação, Ciência e Tecnologia do Rio Grande do Sul. Projeto Político-Pedagógico do Mestrado Profissional em Informática na Educação, 2014. Disponível em: < http://bit.ly/2LW5AmH>. Acesso em 12 set 2019.

LAKATOS, E. M.; MARCONI, M. A. Fundamentos de Metodologia Científica. 6. ed. São Paulo: Atlas, 2006.

MELO, M. V. Três décadas de Pesquisa em Educação Matemática na Unicamp: Um Estudo Histórico a partir de Teses e Dissertações. 230 f. Dissertação (Mestrado em 460 Educação) - Faculdade de Educação, Universidade Estadual de Campinas UNICAMP, Campinas. 2006. Disponível em: < http://bit.ly/2JBQmSp>. Acesso em: 12 set 2019.

MORAES, R.; GALIAZZI, M. C. Análise Textual Discursiva: Processo Reconstrutivo de Múltiplas Faces. Ciência \& Educação, v. 12, n. 1, p. 117-128, 2006. Disponível em: $<$ http://bit.ly/2GeAL9c>. Acesso em: 07 set 2019.

MOROSINI, M. C. Estado de conhecimento e questões do campo científico. Revista Educação. Santa Maria, v. 40, n. 1, p. 101-116, jan./abr. 2015. Disponível em: <http://bit.ly/2xMy89X>. Acesso em: 12 set 2019.

NIEZER, T. M. et al. Caracterização dos Produtos Desenvolvidos por um Programa de Mestrado Profissional da Área de Ensino de Ciências e Tecnologia. Revista Brasileira de Ensino de Ciência e Tecnologia. v. 8, n. 3, mai/ago. 2015. Disponível em: $<$ http://bit.ly/2xNSW0P>. Acesso em 02 set 2019.

OLIVEIRA, M. A.; FERNANDES, M. C. S. G. Formação e produção de conhecimento na universidade: estratégias de graduandos e pós-graduandos para a inserção no campo. Perspectiva. Florianópolis, v. 35, n. 2, p. 658-671, abr./jun. 2017. Disponível em: <http://bit.ly/2xRlKW4>. Acesso em 12 set 2019.

SARTORI, A. S.; SOARES, M. S. P. Concepção dialógica e as NTIC: A educomunicação e os ecossistemas comunicativos. Núcleo de Comunicação e Educação da Universidade de São Paulo, 2013.

SEVERINO, A. J. Educação e universidade: conhecimento e construção da cidadania. Interface. Comunic, Saúde, Educ., v. 6, n. 10, p. 117-24, fev. 2002. Disponível em: $<$ http://bit.ly/2JNQRYn>. Acesso em 13 set 2019. 
VIEIRA, E. Democracia e política social. São Paulo: Cortez, Coleção Polêmicas do nosso tempo, v. 49, 1992. 